\title{
Personalized Type 2 Diabetes Management: An Update on Recent Advances and Recommendations
}

\author{
David M Williams $\mathbb{D}^{\prime}$, Hannah Jones', Jeffrey W Stephens $\mathbb{D}^{1,2}$ \\ 'Department of Diabetes and Endocrinology, Morriston Hospital, Swansea Bay University Health Board, Swansea, SA6 8NL, UK; ${ }^{2}$ Diabetes Research \\ Group, Swansea University Medical School, Swansea University, Swansea, SA2 8PP, UK
}

Correspondence: David M Williams, Diabetes Centre, Morriston Hospital, Swansea, SA6 6NL., UK, Tel +44I792704078,

Email david.williams@doctors.org.uk

\begin{abstract}
Previous guidelines for the treatment of people with type 2 diabetes mellitus (T2D) have relied heavily upon rigid algorithms for the sequential addition of pharmacotherapies to achieve target glycemic control. More recent guidelines advocate a personalized approach for diabetes treatment, to improve patient satisfaction, quality of life, medication adherence and overall health outcomes. Clinicians should work with patients to develop personalized goals for their treatment, including targeted glycemic control, weight management, prevention and treatment of associated comorbidities and avoidance of complications such as hypoglycemia. Factors that affect the intensity of treatment and choice of pharmacotherapy should include medical and patient influences. Medical considerations include the diabetes phenotype, biomarkers including genetic tests, and the presence of comorbidities such as cardiovascular, renal, or hepatic disease. Patient factors include their treatment preference, age and life expectancy, diabetes duration, hypoglycemia fear and unawareness, psychological and social circumstances. The use of a personalized approach in the management of people with T2D can reduce the cost and failure associated with the algorithmic "one-size-fits-all" approach, to anticipate disease progression, improve the response to diabetes pharmacotherapy and reduce the incidence of diabetes-associated complications. Ultimately, the use of personalized medicine in people with T2D should improve medication adherence, patient satisfaction and quality of life to reduce diabetes distress and improve physical health outcomes.
\end{abstract}

Keywords: type 2 diabetes mellitus, personalized management, precision medicine, resources, treatment

\section{What is Personalized Type $\mathbf{2}$ Diabetes Management?}

Currently, the management of type 2 diabetes (T2D) is driven by established international guidelines, and until recent years these did not take account of individual characteristics and the presence of co-morbidities for individual patients. Much of the treatment options recommended by guidelines are based on evidence accumulated from Phase 3 clinical trials and real-world evidence based on population-based studies. These recommendations have clearly made a difference to overall diabetes care. ${ }^{1,2}$ These guidelines do not examine the concept of individualized or personalized management. Individuals differ in their presentation of T2D, some have a short duration, others a long duration and other complications at the time of presentation. Therefore, with respect to treatment, "one size does not fit all". More recently, the American Diabetes Association (ADA) and European Association for the Study of Diabetes (EASD) (along with other guidelines) have recommended tailoring therapy to be more stringent and less stringent based on patients' attitudes, hypoglycemia risk, disease duration, life expectancy, comorbidities and resources. ${ }^{3}$

Personalized diabetes management is based on developing a clinical plan that is tailored to the individual. This may take into account many complex factors. These included patient factors, social, medical (including complications) as well as phenotypic, biochemical and genetic factors (see Figure 1). Therefore, the concept of personalized management is complex and broad. The therapeutic options for managing T2D have increased considerably in the past 10 years, so perhaps the time has come to focus and tailor therapy to the phenotype and personal characteristics of the patient. 


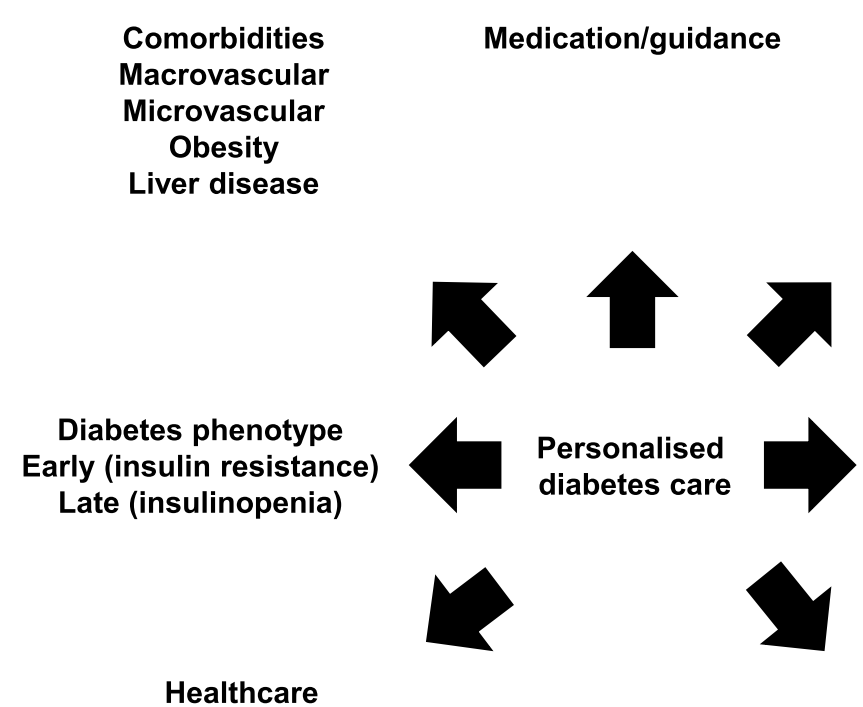

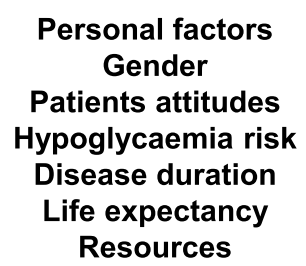

\section{Biochemical markers}

Healthcare

Genetic

resources

factors

Figure I Personalized diabetes care. This figure summarizes the key considerations that are needed when contemplating the choice of diabetes pharmacotherapy for a patient with T2D.

Personalized care may provide the opportunity to address two potential reasons for the continued morbidity and mortality associated with T2D. These include firstly, the suboptimal application of evidence-based therapies (eg, due to lack of medication intensification or insufficient lifestyle changes or medication adherence by patients) and secondly inadequate efficacies of current therapies when optimally applied. ${ }^{4}$

It may be argued that personalized diabetes care has been practiced for many years, ${ }^{5}$ but the complexity of integrating this into the routine management of T2D remains a challenge with many barriers. ${ }^{6}$ To date there are few studies, which have examined the effects of personalized care on diabetes outcome, but evidence does exist to support this approach. ${ }^{7,8}$ This review focuses on examining the personal factors, phenotypic characteristics, biomarkers and genetic markers that may have a role to personalize the management of T2D.

\section{Phenotypic Factors in the Personalized Management of T2D The Diabetes Continuum}

The pathophysiology of T2D is complex. There are three main processes contributing to elevated glucose levels. Insulin resistance results in reduced peripheral glucose utilization in skeletal muscle, adipose tissue and the liver. Excess hepatic glucose release as a result of pancreatic islet dysfunction associated with impaired beta-cell insulin release and excess glucagon release from the alpha-cells contribute to hyperglycemia. Along with this, less insulin release is associated with reduced glucose uptake in the periphery. The situation worsens with time as glucotoxicity and associated inflammation and oxidative stress result in further beta-cell dysfunction and insulin resistance. ${ }^{9,10}$ The relative contributions of these pathophysiological process change over time. Early in the development of impaired glucose tolerance, beta-cell dysfunction and insulin resistance start many years before the diagnosis. At the time of manifest T2D, typically $50 \%$ of beta-cell function has been lost. Thereafter, the degree of beta-cell dysfunction drives hyperglycemia and the associated complications of T2D. ${ }^{11,12}$ The result is that early in the diabetes continuum there is typically hyperinsulinemia and increased insulin secretion, while normoglycemia may be maintained. As insulin sensitivity decreases and pancreatic beta-cell dysfunction ensues, insulin secretion falls and this may still be in the setting of insulin resistance. As a consequence, hyperglycemia develops and the late complications of diabetes. Therefore, typically with a long duration of T2D, there are associated microvascular and macrovascular complications, insulin deficiency associated with beta-cell exhaustion along with/without insulin resistance. ${ }^{13}$ Additional factors that accelerate the development of diabetes-related 


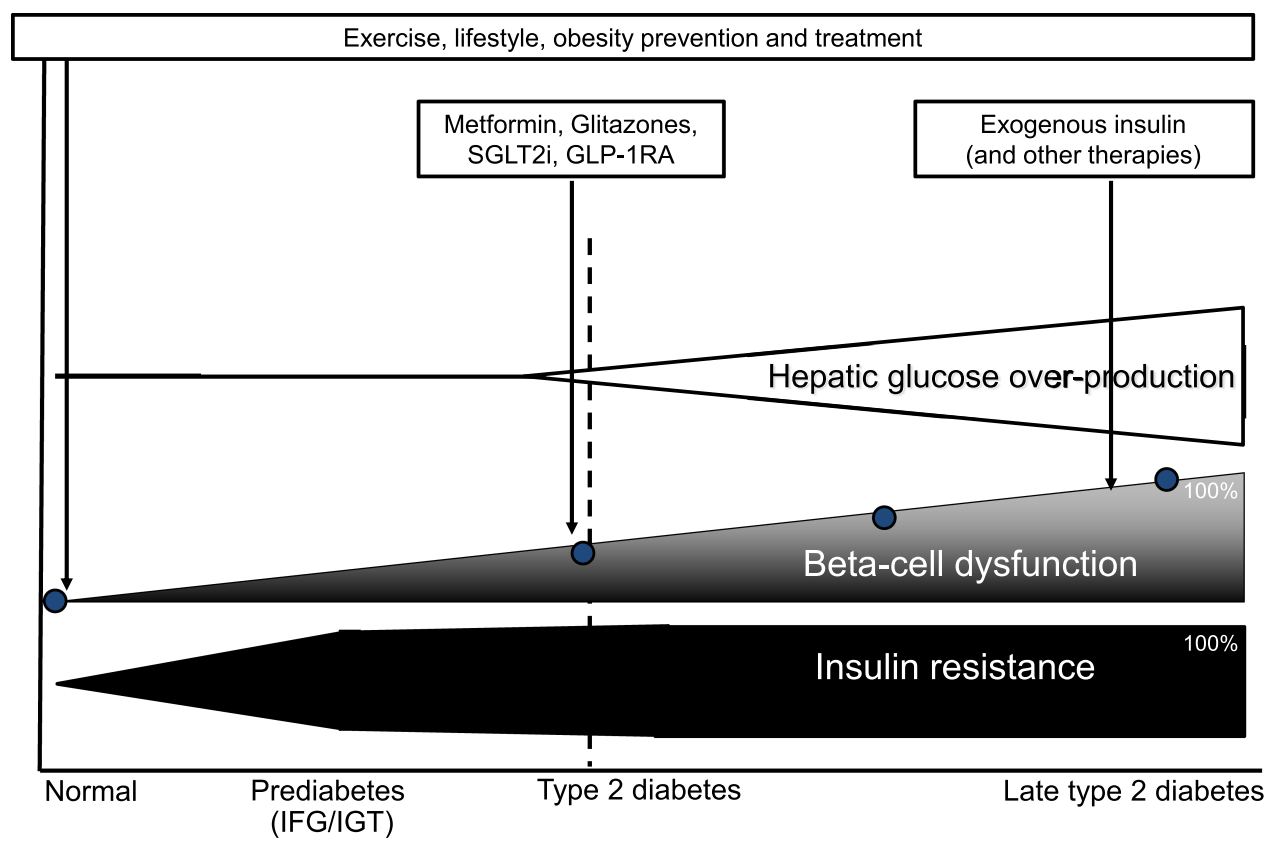

Figure 2 Therapeutic strategies for T2DM during time. This figure summarizes the key therapeutic strategies employed for people with T2D at different stages. Abbreviations: IFG, impaired fasting glucose; IGT, impaired glucose tolerance; SGLT2i, sodium-glucose cotransporter-2 inhibitors; GLP-IRA, glucagon-like peptide-I receptor analogue.

complications include advancing age, obesity, comorbid hypertension and dyslipidemia, smoking status, and genetic factors. $^{14,15}$

Therefore, the management of T2D should be personalized and tailored to the stage that the individual exists within the diabetes continuum. Indeed, some authors have proposed alternative diabetes classification systems based on the degree and rate of decline in beta-cell function, which would guide personalized treatment of diabetes to address insulin resistance or insulin deficiency depending upon the level of beta-cell function. ${ }^{16}$ Prior to the development of T2D, lifestyle, exercise and weight loss are essential to reduce the risk of insulin resistance. Targeting this stage with conservative approaches and subsequent pharmacological and bariatric surgical approaches may be effective. In the individual with impaired glucose tolerance (or prediabetes), the focus should be on methods to reduce insulin resistance. In addition to the management options described above, insulin sensitizers such as metformin and thiazolidinedione may be appropriate. At the point of islet cell dysfunction, therapies addressing insulin and glucagon dysfunction such as glucagon-like peptide-1 receptor analogues (GLP-1RA) would be appropriate. ${ }^{17}$ Lowering glucose toxicity by these means and with the use of sodium-glucose co-transporter 2 inhibitors (SGLT2i) would aid in preserving islet function. Ultimately in the setting of a long duration of diabetes, insulin therapy would be the choice, but there may well be the need for an additional therapy to help address insulin resistance (Figure 2). ${ }^{17}$ People at later stages of the T2D continuum, typically associated with complications, would clearly benefit from multiple therapies with the addition of agents that have a proven benefit in cardiovascular and renal disease, if the patient was not already receiving these (eg, GLP-1RA or SGLT2i). In particular, those with persistent hyperglycemia (eg, HbA1c $\geq 86 \mathrm{mmol} / \mathrm{mol}(10.0 \%)$ ) or those with signs of insulinopenia (eg, ketosis, hypertriglyceridemia) may benefit from the addition of exogenous insulin. ${ }^{17}$

\section{Phenotypic Differences}

As already described, T2D is a heterogeneous condition with respect to pathophysiology but also in relation to clinical course and also among individuals. While it is recognized that individuals presenting with early onset T2D have more rapid progression, ${ }^{18,19}$ there are different phenotypic characteristics, which also influence complication risk. To date, there has been limited effort in identifying phenotypic features that may be associated with increased complication risk and hence targeting individual therapy. 
Table I Phenotypic Characteristics and Prevalence in the ANDIS (All New Diabetics in Scania) Cohort

\begin{tabular}{|c|c|c|}
\hline Subgroup & Phenotypic Characteristics & Prevalence \\
\hline $\begin{array}{l}\text { Severe autoimmune diabetes } \\
\text { (SAID) }\end{array}$ & $\begin{array}{l}\text { Early-onset disease, relatively low BMI, poor metabolic control, insulin deficiency, and presence of } \\
\text { GADA }\end{array}$ & $6.5 \%$ \\
\hline $\begin{array}{l}\text { Severe insulin-deficient } \\
\text { diabetes (SIDD) }\end{array}$ & $\begin{array}{l}\text { Low age at onset, relatively low BMI, low insulin secretion (HOMA2-B index), poor metabolic } \\
\text { control, and GADA negative }\end{array}$ & $17.5 \%$ \\
\hline $\begin{array}{l}\text { Severe insulin-resistant } \\
\text { diabetes (SIRD) }\end{array}$ & Insulin resistance (high HOMA2-IR index) and high BMI & $15.3 \%$ \\
\hline $\begin{array}{l}\text { Mild obesity-related diabetes } \\
\text { (MOD) }\end{array}$ & Obesity but not by insulin resistance & $21.6 \%$ \\
\hline $\begin{array}{l}\text { Mild age-related diabetes } \\
\text { (MARD) }\end{array}$ & Older than patients in other clusters, but modest metabolic derangements & $39.1 \%$ \\
\hline
\end{tabular}

Note: Data from Ahlqvist et al. ${ }^{20}$

Abbreviations: BMI, body mass index; GADA, glutamate decarboxylase antibodies: homeostatic model assessment 2 to estimate beta-cell function (HOMA2-B) and insulin resistance (HOMA2-IR).

\section{Phenotypic Clustering as Groups: Lessons from ANDIS}

The major traditional classification of diabetes has been into type 1 diabetes (T1D) and T2D. While this historically may have been useful to differentiate distinct pathophysiological mechanisms with clear therapeutic implications, it has been insufficient in explaining the wide variety of clinical manifestations associated with diabetes. As discussed, T2D is a heterogeneous condition with different pathophysiological processes. Several clinical questions remain unanswered in our understanding of T2D. For example, we cannot predict the rate of beta-cell failure; why do some patients require aggressive escalation of therapy; what type of medication is best suited for a given patient; why do some patients progress rapidly to microvascular and/or macrovascular complications. ${ }^{20}$ It has been noted that phenotypic subgroups of patients with diabetes can be made based on six factors. These included age at diagnosis, body mass index (BMI), the presence of glutamate decarboxylase antibodies (GADA) and insulin resistance as measured with homeostatic model assessment 2 to estimate beta-cell function (HOMA2-B) and insulin resistance (HOMA2-IR) using C-peptide concentrations. Ahlqvist et al used these variables to create five phenotypic clusters in a sample of approximately 9000 Scandinavian patients with newly diagnosed diabetes (see Table 1). ${ }^{20}$ They observed that with the traditional diagnostic categories, the prevalence of diabetes was T1D 1.2\%, latent autoimmune diabetes in adults $5.2 \%$, and T2D 93.6\%. However, when these were classified according to the predefined phenotypic groups, the prevalence within groups were considerably different, as shown in Table 1. Those belonging to the severe autoimmune diabetes and severe insulindeficient diabetes groups had higher HbA1c levels and these levels remained relatively higher. They also observed that the group with severe insulin-deficient diabetes, had an increased risk of retinopathy and neuropathy, whereas the severe insulin-resistant diabetes group had the highest risk for diabetes-related kidney disease (DKD) and fatty liver, emphasizing the importance of insulin resistance for DKD and hepatosteatosis in T2D. ${ }^{20,21}$ This approach to phenotypic grouping would allow management to be personalized and tailored to the individual, such that those with marked insulin deficiency would commence exogenous insulin therapy early, those with severe insulin resistance could be potentially targeted with insulin sensitizers and others with traditional therapeutic options. Furthermore, education and lifestyle advice could be tailored according to the phenotype.

\section{Geographical Phenotypic Differences in T2D}

Compared to European white people with T2D, people from South Asia differ in their phenotype of T2D with a younger age of onset associated with more marked hyperglycemia, dyslipidemia, nephropathy and cardiovascular disease. Additionally, specific issues may influence treatment such as poor awareness of the disease, a delay in diagnosis, inadequate treatment, and non-compliance with lifestyle recommendations and drug treatment. This has been highlighted by Misra et al. ${ }^{22,23}$ 
With respect to East Asia, biological differences have been identified compared to Caucasians that may increase the risk of T2D and associated complications. ${ }^{24,25}$ Certain East Asian phenotypes have been identified which may be associated with adverse outcomes. These include a low BMI; increased visceral fat; insufficient beta-cell response to counter insulin resistance; high rates of central obesity and the metabolic syndrome; high rates of childhood obesity, gestational diabetes, and renal disease; high rate of young-onset T2D; increased inflammatory markers; low rate of autoimmune T1D; social disparity and psychosocial stress.

\section{Gender Differences}

Little focus has occurred in relation to T2D and gender differences in risk factors, pathophysiology and complications. ${ }^{26}$ While T2D management guidelines recommend individualized therapy based on age, duration of disease, presence of complications and risk of hypoglycemia, the individual's gender has no impact on clinical decisions. Increasing evidence suggests that biological and psychosocial differences between men and women impact diabetes progression and complications. Furthermore, choices and preferences of therapeutic strategies including adherence to lifestyle and pharmacological interventions differ in both genders. ${ }^{27}$ Such differences include differences in hypoglycaemia rates associated with insulin use, differences in the rates of complications and psychological factors relating to depression and stress (for review $\sec ^{27}$ ).

\section{Clinical Factors Influencing Management Options}

When approaching the pharmacological management of patients with T2D, numerous clinical influences need to be considered, which are broadly divided into medical and patient-related factors. Naturally, medical comorbidities such as cardiovascular disease, DKD, liver disease, obesity and hypertension will influence the physician's choice of medication. However, clinical evidence is largely derived from phase 3 trials involving selected participants that do not accurately represent real-life populations. Consequently, there is a mounting sentience of the importance of patient-related influences such as personal preference, age and life expectancy, duration of diabetes, hypoglycemia risk and awareness, and psychosocial factors. Underpinning the choice of pharmacotherapy should be a discussion between the patient and physician about the goals of treatment, and a consideration of the risks and benefits of the various pharmacological options with individualized glycemic targets.

Undoubtedly, while pharmacotherapies used for the treatment of T2D should aim to improve glycaemia and associated medical comorbidities, they should be individualized to be the most acceptable to the patient with a minimal risk of harm. Regrettably, multiple patient and healthcare-related factors adversely impact the realization of target glycemic control. Healthcare factors, which may affect patients, include a lack of integrated care in many health systems and clinical inertia among health professionals. Patient factors may include lower educational attainment, lower income, perceptions around treatment efficacy and the actual impact of T2D on their health ${ }^{28}$ Clearly, these factors ought to be considered when approaching a holistic management strategy in T2D patients.

Over the last decade, guidance from the ADA and EASD, among others, strongly advocates a personalized approach to T2D management. ${ }^{3,17,29,30}$ The medical and personal factors which the physician and patient must consider in this personalized approach are detailed below.

\section{Medical Factors Cardiovascular Disease}

The evaluation of cardiovascular outcomes associated with pharmacotherapies for diabetes has been a hot topic since the rosiglitazone controversy. ${ }^{31}$ Dedicated cardiovascular outcome trials (CVOTs) are now mandatory for diabetes therapies to demonstrate no increased risk of cardiovascular harm associated with their use. However, several therapies have been observed to significantly reduce the risk of 3-point major adverse cardiovascular events (3-P MACE), a composite outcome of nonfatal stroke, nonfatal myocardial infarction, and cardiovascular death. Therapies within the GLP-1RA and SGLT2i classes have the strongest evidence to support cardiovascular benefit in people with T2D. ${ }^{32,33}$ Canagliflozin and empagliflozin demonstrate cardiovascular protection in the SGLT2i class, while dulaglutide, liraglutide and semaglutide improve cardiovascular outcomes in the GLP-1RA class. More recently, efpeglenatide, a newer GLP-1RA, was observed 
Table 2 Summary of Type 2 Therapies: Effects on Cardiovascular Risk, Body Weight, Renal Use and Adverse Effects

\begin{tabular}{|c|c|c|c|c|}
\hline \multirow[t]{2}{*}{ Drug } & \multicolumn{2}{|c|}{ Cardiovascular } & \multirow[t]{2}{*}{ Effect on Body Weight } & \multirow[t]{2}{*}{ Major Side Effects } \\
\hline & 3P MACE & HF & & \\
\hline Biguanide & & & & GI disturbance, lactic acidosis \\
\hline Metformin & Not done & Not done & Weight neutral & \\
\hline Sulphonylurea & & & & Weight gain, hypoglycaemia \\
\hline Gliclazide & Not done & Not done & Weight gain & \\
\hline Glimepiride & Not done & Not done & Weight gain & \\
\hline Thiazolidinedione & & & & Oedema, heart failure, weight gain, fractures \\
\hline Pioglitazone & Not done & Not done & Weight gain & \\
\hline DPP-IV inhibitor & & & & Gl disturbance, pancreatitis (uncommon) \\
\hline Alogliptin ${ }^{36}$ & $\leftrightarrow$ & Not reported & Weight neutral & \\
\hline Saxagliptin ${ }^{37}$ & $\leftrightarrow$ & - & Weight neutral & \\
\hline Sitagliptin ${ }^{38}$ & $\leftrightarrow$ & $\leftrightarrow$ & Weight neutral & \\
\hline Linagliptin 39 & $\leftrightarrow$ & $\leftrightarrow$ & Weight neutral & \\
\hline Vildagliptin & Not done & Not done & Weight neutral & \\
\hline SGLT-2 inhibitor & & & & UTI, genital thrush \\
\hline Canagliflozin ${ }^{40}$ & + & + & Weight loss & \\
\hline Dapagliflozin ${ }^{41,42}$ & $\leftrightarrow$ & + & Weight loss & \\
\hline Empagliflozin $^{43-45}$ & + & + & Weight loss & \\
\hline Ertugliflozin ${ }^{46}$ & $\leftrightarrow$ & + & Weight loss & \\
\hline GLP-IRA & & & & Gl disturbance, pancreatitis (uncommon) \\
\hline Dulaglutide $^{47}$ & + & $\leftrightarrow$ & Weight loss & \\
\hline Exenatide BD (Byetta) & No CVOT & No CVOT & Weight loss & \\
\hline Exenatide QW (Bydureon) ${ }^{48}$ & $\leftrightarrow$ & $\leftrightarrow$ & Weight loss & \\
\hline Liraglutide $^{49}$ & + & $\leftrightarrow$ & Weight loss & \\
\hline Lixisenatide $^{50}$ & $\leftrightarrow$ & $\leftrightarrow$ & Weight loss & \\
\hline Semaglutide ${ }^{51,52}$ & + & $\leftrightarrow$ & Weight loss & \\
\hline Insulin 57,59 & $\leftrightarrow$ & $\leftrightarrow$ & Weight gain & Weight gain, hypoglycaemia \\
\hline
\end{tabular}

Notes: + denotes superiority versus placebo, $\leftrightarrow$ denotes non-inferiority versus placebo, - denotes inferiority versus placebo.

Abbreviations: 3P MACE 3, point major adverse cardiac events; DPP-IV, Dipeptidyl peptidase-4; eGFR, estimated glomerular filtration rate; GI, gastrointestinal; GLP-IRA, glucagon-like peptide-I receptor analogue; HF, heart failure; SGLT-2, sodium-glucose co-transporter-2; UTI, urinary tract infection.

to significantly reduce 3-P MACE in people with T2D and either cardiovascular or renal disease. ${ }^{34}$ However, this drug is not yet approved for use in T2D. Each of the dipeptidyl peptidase-IV (DPP-IV) inhibitors were not associated with cardiovascular harm. ${ }^{35-39}$ Table 2 presents the cardiovascular impact of diabetes pharmacotherapies in the major CVOTs. ${ }^{36-52}$ There have been no prospective placebo-controlled CVOTs for metformin, sulphonylureas, thiazolidinediones or insulin. One meta-analysis reported that metformin reduced the rate of cardiovascular events, cardiovascular mortality and all-cause mortality. ${ }^{53}$ Compared with other treatments, sulphonylurea use in people with T2D was associated with increased cardiovascular and all-cause mortality. ${ }^{54}$ However, a prospective trial comparing the impact of add-on therapy with either glimepiride or linagliptin observed no difference in 3P-MACE. ${ }^{55}$ One meta-analysis reported pioglitazone use in people with pre-diabetes or T2D reduced 3P-MACE, though there was an increased risk of heart failure, edema, weight gain and bone fractures. ${ }^{56}$ While insulin use is usually reserved for those with more advanced or poorly controlled T2D who typically have greater cardiovascular risk, randomized-controlled trials to date have not observed a greater risk of 3-P MACE versus the standard of care. ${ }^{57-59}$

Secondary endpoints of major CVOTs observed improved heart failure outcomes with several therapy classes. Dedicated trials have been undertaken to establish the efficacy of SGLT2i even in people without diabetes. Dapagliflozin reduces the incidence of worsening heart failure in people with heart failure and reduced ejection fraction (HFrEF), irrespective of underlying T2D. ${ }^{42}$ Empagliflozin use in those with $\mathrm{HFrEF}^{44}$ or heart failure with preserved ejection fraction $(\mathrm{HFpEF})^{45}$ is associated with a reduced incidence of worsening heart failure, regardless of underlying T2D. Recently, the license for dapagliflozin has been extended in Europe and the US for the treatment of people with 
HFrEF. Given the improved cardiovascular outcomes associated with empagliflozin in people with $\mathrm{HfpEF}{ }^{45}$ and the anticipated results of the DELIVER study investigating dapagliflozin in people with $\mathrm{HFpEF},{ }^{60}$ it will be interesting to see whether the license for SGLT-2 inhibitors will extend to HFpEF in future. Heart failure outcomes from the major CVOTs to date are presented in Table 2.

\section{Diabetes-Related Kidney Disease (DKD)}

In patients with DKD, clinicians need to regularly review pharmacotherapy for both glycemic control and to reduce the decline in renal function to avoid the need for renal replacement therapy and prevent renal death. The use of some classes of medication in people with DKD should be avoided, either because their use is associated with a greater risk of adverse events or there is diminished efficacy for glycemic control. Conversely, the use of some T2D therapies have good evidence to support their use in DKD to reduce disease progression and ultimately improve patient's duration and quality of life. Renal outcomes associated with diabetes pharmacotherapy are reviewed elsewhere. ${ }^{61,62}$

As discussed, there is enormous interest to evaluate the cardiovascular safety of diabetes pharmacotherapies. Many of the CVOTs to date have demonstrated that use of these medications stabilizes the decline in renal function. ${ }^{61}$ Dedicated trials powered to evaluate renal endpoints have been completed, demonstrating that both canagliflozin ${ }^{63}$ and dapagliflozin ${ }^{64}$ significantly reduce major adverse renal outcomes. Recently, the license for dapagliflozin has been extended in Europe and the US for the prevention of DKD even in those with severe renal impairment and without a diagnosis of T2D.

Secondary outcomes from studies investigating GLP-1RAs have observed a reduced incidence and progression of albuminuria, though there is minimal impact on the decline in eGFR in patients with T2D. ${ }^{61,65}$ The use of DPP-IV inhibitors in people with T2D and DKD is safe, and the dose of linagliptin does not need adjustment even in those with end-stage renal disease (ESRD). However, DPP-IV inhibitors have minimal impact on DKD progression. ${ }^{66}$ Metformin use is contraindicated in people with an estimated glomerular filtration rate (eGFR) $<30 \mathrm{~mL} / \mathrm{min} / 1.73 \mathrm{~m}^{2}$ due to the risk of lactic acidosis. Caution should be exercised with sulphonylureas and thiazolidinediones in people with advanced DKD due to the risk of hypoglycemia and fluid overload. Insulin use in patients with DKD is associated with a greater risk of hypoglycemia and the dose should be titrated carefully. The pharmacological management of T2D in people with DKD is detailed in a further review article. ${ }^{67}$

\section{Non-Alcoholic Fatty Liver Disease}

There is an increasing recognition of the association of non-alcoholic fatty liver disease (NAFLD) and non-alcoholic steatohepatitis (NASH) in people with T2D, and the subsequent risk of cirrhosis, decompensated liver disease and hepatocellular carcinoma. ${ }^{68}$ Current treatment options for NAFLD are limited, and patients are generally advised to undertake dietary and lifestyle measures to aid weight loss and reduce liver fat content. Pioglitazone use is associated with improved glucose control, insulin resistance, liver function tests, and measures of hepatic fat and inflammation, ${ }^{69,70}$ though there is limited evidence of benefit in people with more advanced fibrotic liver disease. Current National Institute for Health Care Excellence (NICE) guidance in the UK supports pioglitazone use in NAFLD. However, there is limited evidence to support their use to reduce major adverse hepatic events such as cirrhosis, decompensated liver disease or hepatocellular carcinoma and studies with longer duration of follow-up are needed to corroborate this.

Recent evidence points towards benefits associated with SGLT2i and GLP-1RA use, which improve liver enzyme measurements in people with T2D. ${ }^{6}$ Indeed, SGLT2i use in people with NAFLD improves biomarkers of liver disease, including liver enzymes, liver fat content and histopathological features (for review see ${ }^{68}$ ). Recently, the SEMA-NASH trial observed NASH resolution in 59\% of the patients receiving $0.4 \mathrm{mg}$ subcutaneous semaglutide daily. ${ }^{71}$ Similarly, use of dulaglutide $1.5 \mathrm{mg}$ weekly in patients with T2D and NAFLD significantly improved liver fat content and liver enzymes. ${ }^{72}$ Unfortunately, there are no longer-term trials to evaluate the impact of SGLT2i or GLP-1RAs on major adverse hepatic outcomes such as cirrhosis, decompensated liver disease or hepatocellular carcinoma and longer followup is needed. Nevertheless, clinicians should consider the use of SGLT2i and/or GLP-1RAs in patients with T2D and NAFLD as current evidence suggests improvements in several biomarkers of liver disease with their use. 


\section{Body Weight}

The growing burden of obesity is concerning, having tripled in prevalence since 1975, and in 2016 there was an estimated 650 million obese adults worldwide (13\% population) and a further 1.9 billion people overweight $(39 \%$ population). In people with $\mathrm{T} 2 \mathrm{D}$, the prevalence of obesity is greater, with some studies estimating the prevalence of patients with obesity at around $50 \%$ and $35 \%$ are overweight. ${ }^{73}$ Obesity is associated with greater all-cause mortality and was associated with over 4 million deaths worldwide in 2015, largely a result of excess cardiovascular disease, T2D and its complications, chronic kidney disease and obesity-associated cancer. ${ }^{74}$ Pharmacotherapy used for the treatment of T2D can impact on changes in body weight, summarized in Table 2. The detailed appraisal on the impact of diabetes treatments on body weight has been reported elsewhere. ${ }^{75}$

The pharmacological options for the treatment of obesity are growing, and GLP-1RAs are one of the modern main players. ${ }^{76}$ These support weight loss by inducing satiety via hypothalamic stimulation and delayed gastric emptying. Liraglutide $1.8 \mathrm{mg}$ daily by subcutaneous injection (Victoza) supports weight loss of 4 to $6 \mathrm{~kg} .{ }^{76}$ Trials evaluating liraglutide $3.0 \mathrm{mg}$ (Saxenda) daily, observed a mean weight loss of $8.4 \mathrm{~kg} .{ }^{77}$ Indeed, Saxenda is licensed for the treatment of obesity even in people without T2D in Europe and the US. Good evidence supports other GLP-1RAs for the treatment of obesity also, especially in those with T2D. Semaglutide $1.0 \mathrm{mg}$ weekly was associated with a mean weight loss of $4.9 \mathrm{~kg}$ in people with T2D. ${ }^{51}$ In a dedicated weight loss trial of overweight and obese patients (with or without T2D), semaglutide $2.4 \mathrm{mg}$ weekly resulted in $15.3 \mathrm{~kg}$ weight loss $\left(14.9 \%\right.$ body weight). ${ }^{78}$ In the REWIND study, dulaglutide was associated with $1.5 \mathrm{~kg}$ weight loss. ${ }^{47}$ While Saxenda remains the only licensed GLP-1RA to treat obesity, weight loss associated with GLP-1RAs should guide the clinician to select these therapies in people with T2D and comorbid obesity. Interestingly, tirzepatide, a combination GLP-1RA and glucose-dependent insulinotropic peptide (GIP) agonist has been observed to have major impact to improve glycaemic control and support weight loss, greater than that observed with other GLP-1RA or basal insulin. ${ }^{79,80}$ However, this combination drug is not yet approved for use in people with T2D.

The use of SGLT2i also supports weight loss in people with T2D, though to a lesser extent. Their use in people with T2D can support an additional weight loss of 2 to $4 \mathrm{~kg}{ }^{76}$ Metformin use is considered at least weight neutral though may be associated with a modest weight loss, as observed in the Diabetes Prevention Program. ${ }^{81}$ The use of DPP-IV inhibitors in people with T2D is considered weight neutral. ${ }^{82}$ The use of sulphonylureas, thiazolidinediones, and insulin are each associated with weight gain and should be avoided (if possible) in patients with T2D who are overweight or obese. ${ }^{83}$

In people with T2D and obesity, bariatric surgery can be considered as a treatment. In the UK, NICE suggest considering referral to bariatric surgical services in people with a diagnosis of T2D less than 10 years and a BMI $>35 \mathrm{~kg} /$ $\mathrm{m}^{2}$. Indeed, a greater rate of remission of T2D is observed in people with a shorter duration of T2D. Bariatric surgery is associated with a range of benefits in people with $\mathrm{T} 2 \mathrm{D}$, including $\mathrm{T} 2 \mathrm{D}$ remission, improved cardiometabolic risk factors, and reduced all-cause mortality. ${ }^{84}$

\section{Blood Pressure}

Hypertension is observed in $50 \%$ to $75 \%$ of people with $\mathrm{T} 2 \mathrm{D},{ }^{85}$ making the use of diabetes therapies to reduce blood pressure (BP) in people with T2D attractive. However, the impact of these medications on BP is modest, and they should not be used as a substitute for anti-hypertensive treatment if BP is uncontrolled. Semaglutide was associated with a reduction in systolic BP (SBP) of $5.4 \mathrm{mmHg}$ in the SUSTAIN-6 CVOT, ${ }^{51}$ with consistent reductions in SBP observed in CVOTs evaluating GLP-1RAs. ${ }^{47-52}$ The use of DPP-IV inhibitors in people with T2D results in a mean SBP reduction of about $3 \mathrm{mmHg} .{ }^{86}$ One meta-analysis also noted that SGLT2i use is associated with a mean SBP reduction of $3.6 \mathrm{mmHg} .{ }^{87}$ While pioglitazone use is associated with a reduced SBP of $3 \mathrm{mmHg}$ in trials, ${ }^{88}$ its use is limited by the associated heart failure risk. Metformin does not significantly impact $\mathrm{BP},{ }^{89}$ and sulphonylureas are associated with a modest SBP rise versus metformin, probably a result of the body weight increase. ${ }^{88}$

\section{Personal Factors}

\section{Personal Preference}

Including patients in the decision-making process of their diabetes treatment is essential and is associated with improved medication adherence, quality of life, healthcare costs and health outcomes. ${ }^{3,28,30}$ Clinicians should be mindful that 
patients with similar personal demographics and medical comorbidities may have very different treatment priorities and preferences. Therefore, having early discussions with patients about their treatment goals is important and fosters the clinician-patient relationship. Patient pharmacotherapy choices and treatment priorities infrequently align with their clinicians, which provides a challenge in management. ${ }^{90}$ Such preferences often involve the route of administration, side effects, driving implications, drug costs, and the effect on health insurance.

\section{Age and Life Expectancy}

Advancing age and frailty are associated with a greater risk of hypoglycemia, and a lesser benefit associated with improved glycemic control. ${ }^{91}$ There is often a lesser value in this patient group associated with the health benefits of improved BP and lipids than the risk of the polypharmacy required to achieve it. This is a result of the greater burden of medical comorbidities observed in those with older age, who frequently have social disadvantages that together heighten the risk and impact of falls, and the potentially catastrophic effects of hypoglycemia.

In patients with older age or reduced life expectancy, a personalized glycemic target acceptable to the patient and clinician to minimize the risk of harm should be agreed. ${ }^{31}$ Consensus guidelines advocate less aggressive glycemic targets in people with T2D in this patient group. There is a general stance to focus on improved quality of life and minimize the risk of acute complications such as hypoglycaemia. ${ }^{3,17,29,30}$ Clinicians should aim whenever possible to avoid drugs which increase the risk of hypoglycemia (sulphonylureas, insulin), are injectable (GLP-1RAs, insulin) or associated with a greater risk of adverse effects (SGLT2i, GLP-1RAs). Physicians should frequently review the benefits of antihypertensive and statin therapy (among other medicines) to avoid the risks and unnecessary healthcare costs of polypharmacy. In contrast, patients with a shorter duration of diabetes and longer life expectancy should have more stringent glycemic, BP and lipid profile targets to reduce the incidence of vascular complications.

\section{Duration of Diabetes}

Patients with a greater duration of T2D will be further along the diabetes continuum and more likely to have pancreatic beta-cell exhaustion, rendering them insulin deficient. These patients are also more likely to have associated microvascular, macrovascular, and other T2D complications, and therefore a more complicated diabetes treatment regime. ${ }^{92}$ Given the higher risk of a catastrophic event secondary to hypoglycemia in patients with cardiovascular disease, patients with a greater duration of T2D are more likely to benefit from less aggressive glycemic targets. ${ }^{29}$ Nonetheless, given the relative degree of beta-cell dysfunction compared to patients with a shorter duration of T2D, they are also more likely to need insulin than those with a shorter T2D duration ${ }^{92}$ and cautious glucose targets should be considered in agreement with the patient. ${ }^{29}$

\section{Hypoglycemia Risk and Awareness}

As discussed, patients with advancing age, greater medical comorbidity, longer duration of diabetes and polypharmacy are at greater risk of hypoglycemia. Hypoglycemia is typically associated with adrenergic symptoms (sweating, tremors, hunger) and neuroglycopenic symptoms (behavioral changes, cognitive impairment, seizures). Hypoglycemia unawareness (HU) is defined as the onset of neuroglycopenic symptoms before adrenergic symptoms or the failure to sense the fall of glucose levels below normal limits. Patients with a longer duration of diabetes, greater age, frequent hypoglycemia or well-controlled glycaemia are more likely to have HU. ${ }^{93}$ While HU is more common in people with T1D, around $10 \%$ of the T2D patients treated with insulin are affected. ${ }^{94}$

Naturally, patients with HU are at greater risk of major adverse events including severe hypoglycemia and sudden death, in addition to a greater risk of neurological, cardiovascular and retinal damage over time resulting in significant morbidity and mortality. ${ }^{93}$ To minimize the risk of severe hypoglycemia and potentially restore hypoglycemia awareness, such patients should have a higher HbAlc target for at least several months. ${ }^{29}$ Additionally, the clinician should counsel the patient on the implications of HU on driving, sleep, employment, exercise and travel for their safety and the safety of others. ${ }^{93}$ Whenever possible, the clinician should adjust the diabetes pharmacotherapy to suit the patients' intended treatment goals and avoid medications with the greatest risk of hypoglycemia, such as sulphonylureas and insulin. ${ }^{3,17,29,30}$ 


\section{Psychosocial Factors}

Psychological problems are common in people with T2D, including greater rates of depression, cognitive impairment, and other psychiatric illness. ${ }^{95}$ When considering diabetes pharmacotherapies for these patients, it is essential to consider the additional stress and challenges associated with injectable therapies and the additional glucose monitoring required for some diabetes treatments. Working with patients to develop personalized treatment priorities including glycemic targets and pharmacotherapy is important. For example, such patients may have erratic glycemic control increasing the risk of hypoglycemia or have psychological distress associated with weight gain, which will obviously influence the physician's pharmacotherapy choice. Clearly, working with patients to find the most acceptable treatment to benefit their diabetes treatment will improve medication adherence and patient satisfaction, and minimize diabetes distress. Nevertheless, it is important for the physician to also ensure that appropriate psychological therapies and support from appropriate services are in place to support patients and treat comorbid psychiatric illness. This is a particular challenge, as patients need to be treated by multiple services that therefore need to work together to improve both physical and psychological outcomes. Needle phobias are also a common barrier to the use of injectable therapies in people with T2D. Before excluding the use of such therapies in people with a needle phobia, clinicians should explore these anxieties to dispel any misconceptions around their use, and approaches that may improve needle acceptability and thereby medication adherence and patient outcomes. ${ }^{96}$

Patients with advanced frailty, cognitive impairment and/or poor social support may be unable to self-administer injectable therapies or undertake sufficient glucose monitoring. In such cases, clinicians should avoid such therapies until necessary, and when required utilize the minimum number of injections and the lowest dose required to achieve target glycemic control. This should be agreed with the patient, their family and/or community nurses as appropriate. . $^{37,29,30}$

\section{Genetic Factors}

During the past decade, considerable research has been undertaken to investigate the genetics of T2D. One of the long-term goals of personalized medicine is to identify genetic markers, which may allow therapies to be tailored to the individual. However, one of the challenges in this field is that there may be many different genes and further genetic variants, which influence the T2D phenotype. The picture is again complex and the "strength" of genetics in identifying responders and nonresponders to various therapies is complicated. While there is an association between genetic makeup and treatment response to diabetes therapies in individual patients, few studies have observed strong pharmacogenomics in this respect. ${ }^{97}$ The best evidence for this approach comes from the treatment of patients with monogenic diabetes, as these subgroups are clearly defined by molecular genetics and demonstrate an excellent response to specific drug therapies. In contrast, T2D is often polygenic and influenced by several environmental factors, which complicate the picture. ${ }^{98,99}$

\section{Monogenic Diabetes}

The application of personalized medicine in people with monogenic forms of diabetes is evidence-based, and practical given the clear genetic definitions of these forms of diabetes and a good example of personalized medicine given the reliable response to treatment dependent upon the underlying genetic defect. ${ }^{98,99}$ Maturity-onset diabetes of the young (MODY) typically occurs in families with several generations in which diabetes develops as young adults with an autosomal dominant inheritance pattern and is frequently misdiagnosed as T2D. ${ }^{99,100}$ With respect to MODY, multiple genes have been implicated in its development including glucokinase $(G C K)$, hepatocyte nuclear factor $(H N F) 1 A$, $H N F 4 A$ and $H N F 1 B$ amongst many others. While the presence of these inherited gene mutations associates with a variety of clinical features, they also associate with different treatment responses and the need for drug intervention at all. For example, patients with $G C K$-MODY typically do not require any treatment (except during pregnancy), whereas patients with $H N F 1 A$-MODY or $H N F 4 A$-MODY typically exhibit an excellent response to sulphonylurea therapy. Similarly, neonatal diabetes mellitus (NDM) is caused by specific gene mutations, and the genetic focus of the mutation can predict the clinical response to different drug therapies. Patients with $A B C C 8$-NDM or KCNJ11-NDM usually respond to sulphonylurea therapy, while those with INS-NDM mutations need early intensive insulin treatment and patients with 
6q24-NDM mutations usually do not require treatment until later in life. ${ }^{98,99}$ Similar, though relatively undefined associations between specific genetic mutations and treatment responses have been observed in other forms of monogenic diabetes including lipodystrophies and severe insulin resistance syndromes.

\section{Polygenic Diabetes}

Of course, most people with diabetes do not have a monogenic form of diabetes. Indeed, recent progress in this area of genomics utilizing genome-wide association studies (GWAS) has identified more than 400 genetic variants associated with the development of T2D, each with a small effect on the net phenotype. ${ }^{101,102}$ These genetic factors are mostly common genetic variants, and the relative significance of each is difficult to establish. This is complicated by the interplay of these genetic markers and the lifestyle and environmental features, which typically associate, with the development of T2D. This fundamentally hinders defining specific etiologies of T2D on a genetic basis, making the use of the clinical parameters discussed previously more attractive and viable to guide treatment options.

Despite the challenges associated with the interpretation of genetic variation and the development of T2D, the potential for personalized treatments based on specific subgroups of T2D utilizing genetic biomarkers is attractive. This may permit the earlier use of more effective therapies for patients to reduce diabetes-related complications and utilize therapies with the lowest risk of adverse events. Given the limited use of individual genetic variants, there is growing interest in the use of genetic risk scores to support diagnosis and treatment of T2D. ${ }^{103}$ Indeed, some studies have found that patients with T2D and a higher genetic burden are more likely to benefit from some treatments such as sulphonylurea therapy. ${ }^{104}$ The use of these genetic risk scores is not sufficiently validated or available to support clinicians in current clinical practice, though increasing interest in this area indicates that there may be a greater role for genetic testing in people with $\mathrm{T} 2 \mathrm{D}$ in the future.

\section{Other Biomarkers}

The use of several biomarkers to support diagnosis and treatment of diabetes are already used in clinical practice, such as antibody testing (eg, GADA, anti-tyrosine phosphatase [anti-IA-2], islet cell autoantibodies [ICA]), and urinary C-peptide to distinguish between T1D and T2D. This is often a problem encountered in the management of T2D, as patients may be initially misdiagnosed and have underlying autoimmune pathology such as T1D or latent autoimmune diabetes in adults (LADA). Higher antibody titers predict a greater likelihood of underlying autoimmune diabetes and therefore the need for insulin therapy now or in future. Lower urinary C-peptide levels suggest lower endogenous insulin secretion and therefore greater need for exogenous insulin therapy. Depending on the clinical picture, the urinary C-peptide level can help to distinguish T1D from T2D or place a patient with T2D at the later stages of the diabetes continuum, suggesting the need for insulin therapy. ${ }^{105}$ Indeed, the presence (or absence) of these biomarkers associates with the response to non-insulin- and insulin-based therapies for T2D, and often guides personalized treatment choices already in current clinical practice. ${ }^{105,106}$

Table 3 An Empirical View of Personalized Care for a Patient with T2DM

\begin{tabular}{|l|}
\hline Current Consideration \\
\hline Does the available guidance apply to this individual? \\
Has the management plan been optimized as per guidance? \\
Does the therapy need to be modified for personalized reasons? \\
Optimize therapy according to clinical phenotype \\
Optimize therapy for medical comorbidity (eg, cardiovascular, renal disease) \\
Are there biomarkers (eg, urinary C-peptide, GADA) to tailor therapy? \\
Are there genetic markers to tailor therapy (eg, MODY testing)? \\
\hline Future considerations \\
\hline Are there biomarkers to personalize therapy? \\
Are there genetic markers to personalize therapy?
\end{tabular}


Additional biomarkers have been observed to associate with the risk of developing T2D in clinical research settings. These biomarkers include fasting proinsulin, total triglycerides, fasting fatty acids, interleukin 1 receptor antagonist, apolipoprotein B/low-density lipoprotein cholesterol ratio, and high sensitivity c-reactive protein, among others. ${ }^{107}$ Nevertheless, the current utility of these other biomarkers in current routine clinical practice is unclear and their use is not routinely recommended. However, use of some of these biomarkers in profiling scores in combination with clinical biomarkers such as BMI or medical comorbidity and genetic biomarkers may in future guide treatment choices in people with T2D.

\section{Conclusion}

The personalization of T2D management has gained precedence in more recent guidance and should account for several medical and personal factors. Key medical factors, which should usually be considered, include the diabetes phenotype, available biomarkers (eg, autoantibodies, urinary C-peptide and genetic tests) and the presence of medical comorbidities such as cardiovascular or renal disease. These are summarized in Table 3. Treatment decisions should also account for presence of other complications such as peripheral vascular disease, retinopathy and neuropathy, which are not discussed in this review. Increasingly, the importance of patient factors such as treatment preference, age and life expectancy, diabetes duration, hypoglycemia fear and unawareness and psychosocial concerns are considered important by the physician when planning a holistic approach to patient management. In future, profiling scores including clinical, biochemical, and genetic variables are likely to impact upon the physician treatment preferences though clearly further research is needed in this are before its clinical utility can be established.

\section{Disclosure}

The authors report no conflicts of interest in this work.

\section{References}

1. Hoerger TJ, Zhang P, Segel JE, et al. Improvements in risk factor control among persons with diabetes in the United States: evidence and implications for remaining life expectancy. Diabetes Res Clin Pr. 2009;86:225-232. doi:10.1016/j.diabres.2009.09.017

2. Wang J, Geiss LS, Cheng YLJ, et al. Long-term and recent progress in blood pressure levels among us adults with diagnosed diabetes, 19882008. Diabetes Care. 2011;34:1579-1581. doi:10.2337/dc11-0178

3. Inzucchi SE, Bergenstal RM, Buse JB, et al. Management of hyperglycemia in type 2 diabetes: a patient-centered approach. Diabetes Care. 2012;35(6):1364-1379. doi:10.2337/dc12-0413

4. Grant RW, Wexler DJ. Personalized medicine in Type 2 diabetes: what does the future hold? Diabetes Manag (Lond). 2012;2:199-204. doi: $10.2217 / \mathrm{dmt} .12 .15$

5. Kerr EA, Heisler M, Krein SL, et al. Beyond comorbidity counts: how do comorbidity type and severity influence diabetes patients' treatment priorities and self-management? J Gen Intern Med. 2007;22:1635-1640. doi:10.1007/s11606-007-0313-2

6. Gillman MW, Hammond RA. Precision treatment and precision prevention integrating "Below and Above the Skin". JAMA Pediatr. 2016;170:9-10. doi:10.1001/jamapediatrics.2015.2786

7. Pinto LC, Rados DV, Remonti LR, et al. Patient-centered management of type 2 diabetes mellitus based on specific clinical scenarios: systematic review, meta-analysis and trial sequential analysis. J Clin Endocr Metab. 2020;105:3588-3599. doi:10.1210/clinem/dgaa534

8. Boels AM, Hart HE, Rutten GE, Vos RC. Personalised treatment targets in type 2 diabetes patients: the Dutch approach. Prim Care Diabetes. 2017;11:71-77. doi:10.1016/j.pcd.2016.08.001

9. Porte D, Kahn SE. The key role of islet dysfunction in type-II diabetes-mellitus. Clin Invest Med. 1995;18:247-254.

10. Inzucchi SE. Oral antihyperglycemic therapy for type 2 diabetes - Scientific review. JAMA-J Am Med Assoc. 2002;287:360-372. doi:10.1001/ jama.287.3.360

11. Gastaldelli A, Ferrannini E, Miyazaki Y, Matsuda M, DeFronzo RA. Beta-cell dysfunction and glucose intolerance: results from the San Antonio metabolism (SAM) study. Diabetologia. 2004;47:31-39. doi:10.1007/s00125-003-1263-9

12. Del Prato S, Marchetti P. Beta- and alpha-cell dysfunction in type 2 diabetes. Horm Metab Res. 2004;36:775-781. doi:10.1055/s-2004-826163

13. Bailey CJ, Day C. Avandamet: combined metformin-rosiglitazone treatment for insulin resistance in type 2 diabetes. Int J Clin Pract. 2004;58:867-876. doi:10.1111/j.1742-1241.2004.00318.x

14. Sheleme T, Mamo G, Melaku T, Sahilu T. Prevalence, patterns and predictors of chronic complications of diabetes mellitus at a large referral hospital in Ethiopia: a prospective observational study. Diabetes Metab Syndr. 2020;13:4909-4918. doi:10.2147/DMSO.S281992

15. Arambewela MH, Somasundaram NP, Jayasekara HBPR, et al. Prevalence of chronic complications, their risk factors, and the cardiovascular risk factors among patients with type 2 diabetes attending the diabetic clinic at a tertiary care hospital in Sri Lanka. J Diabetes Res. 2018;2018:1-10. doi:10.1155/2018/4504287

16. Schwartz SS, Epstein S, Corkey BE, et al. The time is right for a new classification system for diabetes: rationale and implications of the beta-cell-centric classification schema. Diabetes Care. 2016;39:179-186. doi:10.2337/dc15-1585

17. American Diabetes Association. 9. Pharmacologic approaches to glycemic treatment: standards of medical care in diabetes-2021. Diabetes Care. 2021;44:S111-S24. doi:10.2337/dc21-S009 
18. Wong J, Molyneaux L, Constantino M, Twigg SM, Yue DK. Timing is everything: age of onset influences long-term retinopathy risk in type 2 diabetes, independent of traditional risk factors. Diabetes Care. 2008;31:1985-1990. doi:10.2337/dc08-0580

19. Hillier TA, Pedula KL. Complications in young adults with early-onset type 2 diabetes - Losing the relative protection of youth. Diabetes Care. 2003;26:2999-3005. doi:10.2337/diacare.26.11.2999

20. Ahlqvist E, Storm P, Karajamaki A, et al. Novel subgroups of adult-onset diabetes and their association with outcomes: a data-driven cluster analysis of six variables. Lancet Diabetes Endo. 2018;6:361-369. doi:10.1016/S2213-8587(18)30051-2

21. Ahlqvist E, Prasad RB, Groop L. Subtypes of type 2 diabetes determined from clinical parameters. Diabetes. 2020;69(10):2086-2093. doi:10.2337/dbi20-0001

22. Misra A, Sattar N, Tandon N, et al. Clinical management of type 2 diabetes in South Asia. Lancet Diabetes Endo. 2018;6:979-991. doi:10.1016/ S2213-8587(18)30199-2

23. Shrivastava U, Misra A. Need for ethnic-specific guidelines for prevention, diagnosis, and management of type 2 diabetes in South Asians. Diabetes Technol The. 2015;17:435-439. doi:10.1089/dia.2014.0213

24. Chan JCN, Yeung R, Luk A. The Asian diabetes phenotypes: challenges and opportunities. Diabetes Res Clin Pr. 2014;105:135-139. doi:10.1016/j.diabres.2014.05.011

25. Lim LL, Tan ATB, Moses K, Rajadhyaksha V, Chan SP. Place of sodium-glucose cotransporter-2 inhibitors in East Asian subjects with type 2 diabetes mellitus: insights into the management of Asian phenotype. $J$ Diabetes Complicat. 2017;31:494-503. doi:10.1016/j. jdiacomp.2016.10.008

26. Kautzky-Willer A, Harreiter J, Pacini G. Sex and gender differences in risk, pathophysiology and complications of Type 2 diabetes mellitus Endocr Rev. 2016;37:278-316. doi:10.1210/er.2015-1137

27. Kautzky-Willer A, Harreiter J. Sex and gender differences in therapy of type 2 diabetes. Diabetes Res Clin Pr. 2017;131:230-241. doi:10.1016/ j.diabres.2017.07.012

28. Polonsky WH, Henry RR. Poor medication adherence in type 2 diabetes: recognizing the scope of the problem and its key contributors. Patient Prefer Adher. 2016;10:1299-1307. doi:10.2147/PPA.S106821

29. American Diabetes Association. 6. Glycemic targets: standards of medical care in diabetes-2021. Diabetes Care. 2021;44:S73-S84 doi:10.2337/dc21-S006

30. Davies MJ, D'Alessio DA, Fradkin J, et al. Management of hyperglycaemia in type 2 diabetes, 2018. A consensus report by the American Diabetes Association (ADA) and the European Association for the Study of Diabetes (EASD). Diabetologia. 2018;61:2461-2498. doi:10.1007/ s00125-018-4729-5

31. Nissen SE, Wolski K. Effect of rosiglitazone on the risk of myocardial infarction and death from cardiovascular causes. New Engl J Med. 2007;356:2457-2471. doi:10.1056/NEJMoa072761

32. Andreasen CR, Andersen A, Knop FK, Vilsboll T. Understanding the place for GLP-1RA therapy: translating guidelines for treatment of type 2 diabetes into everyday clinical practice and patient selection. Diabetes Obes Metab. 2021;23:40-52. doi:10.1111/dom.14500

33. Li CX, Liang S, Gao LY, Liu H. Cardiovascular outcomes associated with SGLT-2 inhibitors versus other glucose-lowering drugs in patients with type 2 diabetes: a real-world systematic review and meta-analysis. PLoS One. 2021;16:e0244689.

34. Gerstein HC, Sattar N, Rosenstock J, et al. Cardiovascular and renal outcomes with efpeglenatide in type 2 diabetes. New Engl J Med. 2021;385:896-907. doi:10.1056/NEJMoa2108269

35. Williams R, de Vries F, Kothny W, et al. Cardiovascular safety of vildagliptin in patients with type 2 diabetes: a European multi-database, non-interventional post-authorization safety study. Diabetes Obes Metab. 2017;19:1473-1478. doi:10.1111/dom.12951

36. White WB, Cannon CP, Heller SR, et al. Alogliptin after acute coronary syndrome in patients with type 2 diabetes. New Engl J Med. 2013;369:1327-1335. doi:10.1056/NEJMoa1305889

37. Scirica BM, Bhatt DL, Braunwald E, et al. Saxagliptin and cardiovascular outcomes in patients with type 2 diabetes mellitus. New Engl J Med. 2013;369:1317-1326. doi:10.1056/NEJMoa1307684

38. Green JB, Bethel MA, Armstrong PW, et al. Effect of sitagliptin on cardiovascular outcomes in type 2 diabetes. New Engl J Med. 2015;373:232-242.

39. Rosenstock J, Perkovic V, Johansen OE, et al. Effect of linagliptin vs placebo on major cardiovascular events in adults with type 2 diabetes and high cardiovascular and renal risk the CARMELINA Randomized Clinical Trial. JAMA-J Am Med Assoc. 2019;321:69-79. doi:10.1001/ jama.2018.18269

40. Neal B, Perkovic V, Matthews DR. Canagliflozin and cardiovascular and renal events in type 2 diabetes reply. New Engl J Med. 2017;377:2099. doi:10.1056/NEJMoa1611925

41. Wiviott SD, Raz I, Sabatine MS. Dapagliflozin and cardiovascular outcomes in type 2 diabetes reply. New Engl J Med. 2019;380:1881-1882. doi:10.1056/NEJMoa1812389

42. McMurray JJV, Solomon SD, Inzucchi SE, et al. Dapagliflozin in patients with heart failure and reduced ejection fraction. New Engl J Med. 2019;381:1995-2008. doi:10.1056/NEJMoa1911303

43. Zinman B, Wanner C, Lachin JM, et al. Empagliflozin, cardiovascular outcomes, and mortality in type 2 diabetes. New Engl J Med. 2015;373:2117-2128. doi:10.1056/NEJMoa1504720

44. Packer M, Anker SD, Butler J, et al. Cardiovascular and renal outcomes with empagliflozin in heart failure. New Engl J Med. 2020;383:1413-1424. doi:10.1056/NEJMoa2022190

45. Anker SD, Butler J, Filippatos G, et al. Empagliflozin in heart failure with a preserved ejection fraction. New Engl J Med. 2021;385:1451-1461. doi:10.1056/NEJMoa2107038

46. Cannon CP, Pratley R, Dagogo-Jack S, et al. Cardiovascular outcomes with ertugliflozin in type 2 diabetes. New Engl J Med. 2020;383:1425-1435. doi:10.1056/NEJMoa2004967

47. Gerstein HC, Colhoun HM, Dagenais GR, et al. Dulaglutide and cardiovascular outcomes in type 2 diabetes (REWIND): a double-blind, randomised placebo-controlled trial. Lancet. 2019;394:121-130. doi:10.1016/S0140-6736(19)31149-3

48. Holman RR, Bethel MA, Mentz RJ, et al. Effects of once-weekly exenatide on cardiovascular outcomes in type 2 diabetes. New Engl J Med. 2017;377:1228-1239. doi:10.1056/NEJMoa1612917 
49. Marso SP, Daniels GH, Brown-Frandsen K, et al. Liraglutide and cardiovascular outcomes in type 2 diabetes. New Engl J Med. 2016;375:311-322. doi:10.1056/NEJMoa1603827

50. Pfeffer MA, Claggett B, Diaz R, et al. Lixisenatide in patients with type 2 diabetes and acute coronary syndrome. New Engl J Med. 2015;373:2247-2257. doi:10.1056/NEJMoa1509225

51. Marso SP, Bain SC, Consoli A, et al. Semaglutide and cardiovascular outcomes in patients with type 2 diabetes. New Engl J Med. 2016;375:1834-1844. doi:10.1056/NEJMoa1607141

52. Husain M, Birkenfeld AL, Donsmark M, et al. Oral semaglutide and cardiovascular outcomes in patients with type 2 diabetes. New Engl J Med. 2019;381:841-851. doi:10.1056/NEJMoa1901118

53. Han YC, Xie HZ, Liu YT, et al. Effect of metformin on all-cause and cardiovascular mortality in patients with coronary artery diseases: a systematic review and an updated meta-analysis. Cardiovasc Diabetol. 2019;18. doi:10.1186/s12933-019-0900-7

54. Bain S, Druyts E, Balijepalli C, et al. Cardiovascular events and all-cause mortality associated with sulphonylureas compared with other antihyperglycaemic drugs: a Bayesian meta-analysis of survival data. Diabetes Obes Metab. 2017;19:329-335. doi:10.1111/dom.12821

55. Rosenstock J, Kahn SE, Johansen OE. Effect of linagliptin vs glimepiride on major adverse cardiovascular outcomes in patients with type 2 diabetes: the CAROLINA Randomized Clinical Trial. JAMA-J Am Med Assoc. 2019;322:2138. doi:10.1001/jama.2019.13772

56. Liao HW, Saver JL, Wu YL, et al. Pioglitazone and cardiovascular outcomes in patients with insulin resistance, pre-diabetes and type 2 diabetes: a systematic review and meta-analysis. BMJ Open. 2017;7:e013927. doi:10.1136/bmjopen-2016-013927

57. Gerstein HC, Bosch J, Dagenais GR, et al. Basal insulin and cardiovascular and other outcomes in dysglycemia. New Engl J Med. 2012;367:319-328.

58. Mannucci E, Ferrannini E. Cardiovascular safety of insulin: between real-world data and reality. Diabetes Obes Metab. 2017;19:1201-1204. doi:10.1111/dom.12967

59. Marso SP, McGuire DK, Zinman B, et al. Efficacy and safety of degludec versus glargine in type 2 diabetes. New Engl J Med. 2017;377:723-732. doi:10.1056/NEJMoa1615692

60. Solomon SD, de Boer RA, DeMets D, et al. Dapagliflozin in heart failure with preserved and mildly reduced ejection fraction: rationale and design of the DELIVER trial. Eur J Heart Fail. 2021;23:1217-1225. doi:10.1002/ejhf.2249

61. Williams DM, Nawaz A, Evans M. Renal outcomes in type 2 diabetes: a review of cardiovascular and renal outcome trials. Diabetes Ther. 2020;11:369-386. doi:10.1007/s13300-019-00747-3

62. de Bhailis A, Azmi S, Kalra PA. Diabetic kidney disease: update on clinical management and non-glycaemic effects of newer medications for type 2 diabetes. Ther Adv Endocrinol Metab. 2021;12::20420188211020664. doi:10.1177/20420188211020664

63. Perkovic V, Jardine MJ, Neal B, et al. Canagliflozin and renal outcomes in type 2 diabetes and nephropathy. New Engl J Med. 2019;380:2295-2306. doi:10.1056/NEJMoa1811744

64. Heerspink HJL, Stefansson BV, Correa-Rotter R, et al. Dapagliflozin in patients with chronic kidney disease. New Engl J Med. 2020;383:1436-1446. doi:10.1056/NEJMoa2024816

65. Yin WL, Bain SC, Min TZ. The effect of glucagon-like peptide-1 receptor agonists on renal outcomes in type 2 diabetes. Diabetes Ther. 2020;11:835-844. doi:10.1007/s13300-020-00798-x

66. Williams DM, Nawaz A, Evans M. Sodium-Glucose Co-Transporter 2 (SGLT2) inhibitors: are they all the same? A narrative review of cardiovascular outcome trials. Diabetes Ther. 2021;12:55-70. doi:10.1007/s13300-020-00951-6

67. Triozzi JL, Gregg LP, Virani SS, Navaneethan SD. Management of type 2 diabetes in chronic kidney disease. BMJ Open Diab Res Ca. 2021;9: e002300.

68. Vincent RK, Williams DM, Evans M. A look to the future in non-alcoholic fatty liver disease: are glucagon-like peptide-1 analogues or sodium-glucose co-transporter-2 inhibitors the answer? Diabetes Obes Metab. 2020;22:2227-2240. doi:10.1111/dom.14196

69. Cusi K, Orsak B, Bril F, et al. Long-term pioglitazone treatment for patients with nonalcoholic steatohepatitis and prediabetes or type 2 diabetes mellitus A Randomized Trial. Ann Intern Med. 2016;165:305-+. doi:10.7326/M15-1774

70. Lian JX, Fu JF. Pioglitazone for NAFLD patients with prediabetes or type 2 diabetes mellitus: a meta-analysis. Front Endocrinol. $2021 ; 12$. doi:10.3389/fendo.2021.615409

71. Newsome PN, Buchholtz K, Cusi K, et al. A placebo-controlled trial of subcutaneous semaglutide in nonalcoholic steatohepatitis. New Engl $J$ Med. 2021;384:1113-1124. doi:10.1056/NEJMoa2028395

72. Kuchay MS, Krishan S, Mishra SK, et al. Effect of dulaglutide on liver fat in patients with type 2 diabetes and NAFLD: randomised controlled trial (D-LIFT trial). Diabetologia. 2020;63:2434-2445. doi:10.1007/s00125-020-05265-7

73. Daousi C, Casson IF, Gill GV, et al. Prevalence of obesity in type 2 diabetes in secondary care: association with cardiovascular risk factors. Postgrad Med J. 2006;82:280-284. doi:10.1136/pmj.2005.039032

74. Afshin A, Forouzanfar MH, Reitsma MB, et al. Health effects of overweight and obesity in 195 countries over 25 years. New Engl JMed. 2017;377:13-27.

75. Apovian CM, Okemah J, O’Neil PM. Body weight considerations in the management of type 2 diabetes. Adv Ther. 2019;36:44-58. doi:10.1007/ s12325-018-0824-8

76. Williams DM, Nawaz A, Evans M. Drug therapy in obesity: a review of current and emerging treatments. Diabetes Ther. 2020;11:1199-1216. doi:10.1007/s13300-020-00816-y

77. Pi-Sunyer X, Astrup A, Fujioka K, et al. A randomized, controlled trial of $3.0 \mathrm{mg}$ of liraglutide in weight management. New Engl J Med. 2015;373:11-22. doi:10.1056/NEJMoa1411892

78. Wilding JPH, Batterham RL, Calanna S, et al. Once-weekly semaglutide in adults with overweight or obesity. New Engl J Med. 2021;384:989-1002. doi:10.1056/NEJMoa2032183

79. Ludvik B, Giorgino F, Jodar E, et al. Once-weekly tirzepatide versus once-daily insulin degludec as add-on to metformin with or without SGLT2 inhibitors in patients with type 2 diabetes (SURPASS-3): a randomised, open-label, parallel-group, phase 3 trial. Lancet. 2021;398:583-598. doi:10.1016/S0140-6736(21)01443-4

80. Frias JP, Nauck MA, Van J, et al. Efficacy and safety of LY3298176, a novel dual GIP and GLP-1 receptor agonist, in patients with type 2 diabetes: a randomised, placebo-controlled and active comparator-controlled Phase 2 trial. Lancet. 2018;392:2180-2193. doi:10.1016/S01406736(18)32260-8 
81. Knowler WC, Barrett-Connor E, Fowler SE, et al. Reduction in the incidence of type 2 diabetes with lifestyle intervention or metformin. New Engl J Med. 2002;346:393-403.

82. Gallwitz B. Clinical Use of DPP-4 Inhibitors. Front Endocrinol. 2019;10. doi:10.3389/fendo.2019.00389

83. van Dieren S, Czernichow S, Chalmers J, et al. Weight changes and their predictors amongst 11140 patients with type 2 diabetes in the ADVANCE trial. Diabetes Obes Metab. 2012;14:464-469. doi:10.1111/j.1463-1326.2012.01556.x

84. Koliaki C, Liatis S, Le Roux CW, Kokkinos A. The role of bariatric surgery to treat diabetes: current challenges and perspectives. BMC Endocr Disord. 2017;17. doi:10.1186/s12902-017-0202-6

85. Colosia AD, Palencia R, Khan S. Prevalence of hypertension and obesity in patients with type 2 diabetes mellitus in observational studies: a systematic literature review. Diabetes Metab Syndr Obes. 2013;17:327-338. doi:10.2147/DMSO.S51325

86. Zhang XD, Zhao QY. Effects of dipeptidyl peptidase-4 inhibitors on blood pressure in patients with type 2 diabetes: a systematic review and meta-analysis. J Hypertens. 2016;34:167-175. doi:10.1097/HJH.0000000000000782

87. Georgianos PI, Agarwal R. Ambulatory blood pressure reduction with SGLT-2 inhibitors: dose-response meta-analysis and comparative evaluation with low-dose hydrochlorothiazide. Diabetes Care. 2019;42:693-700. doi:10.2337/dc18-2207

88. Roumie CL, Liu XL, Choma NN, et al. Initiation of sulfonylureas versus metformin is associated with higher blood pressure at one year Pharmacoepidem Dr S. 2012;21:515-523. doi:10.1002/pds.3249

89. Snorgaard O, Kober L, Carlsen J. The effect of metformin on blood pressure and metabolism in nondiabetic hypertensive patients. J Intern Med. 1997;242:407-412. doi:10.1046/j.1365-2796.1997.00236.x

90. Karagiannis T, Avgerinos I, Toumpalidou M, et al. Patients' and clinicians' preferences on outcomes and medication attributes for type 2 diabetes: a mixed-methods study. J Gen Intern Med. 2020. doi:10.1007/s11606-019-05608-0

91. Longo M, Bellastella G, Maiorino MI, et al. Diabetes and aging: from treatment goals to pharmacologic therapy. Front Endocrinol. $2019 ; 10$. doi:10.3389/fendo.2019.00045

92. Hayashino Y, Izumi K, Okamura S, et al. Duration of diabetes and types of diabetes therapy in Japanese patients with type 2 diabetes: the Japan diabetes complication and its prevention prospective study 3 (JDCP study 3). J Diabetes Invest. 2017;8:243-249. doi:10.1111/jdi.12550

93. Martín-Timón I, Del Cañizo-Gómez FJ. Mechanisms of hypoglycemia unawareness and implications in diabetic patients. World J Diabetes. 2015;6:912-926. doi:10.4239/wjd.v6.i7.912

94. Schopman JE, Geddes J, Frier BM. Prevalence of impaired awareness of hypoglycaemia and frequency of hypoglycaemia in insulin-treated Type 2 diabetes. Diabetes Res Clin Pr. 2010;87:64-68. doi:10.1016/j.diabres.2009.10.013

95. Ducat L, Philipson LH, Anderson BJ. The mental health comorbidities of diabetes. JAMA-J Am Med Assoc. 2014;312:691-692. doi:10.1001/ jama.2014.8040

96. Kruger DF, Larue S, Estepa P. Recognition of and steps to mitigate anxiety and fear of pain in injectable diabetes treatment. Diabetes Metab Syndr. 2015;8:49-56. doi:10.2147/DMSO.S71923

97. Zhou KX, Pedersen HK, Dawed AY, Pearson ER. Pharmacogenomics in diabetes mellitus: insights into drug action and drug discovery. Nat Rev Endocrinol. 2016;12:337-346. doi:10.1038/nrendo.2016.51

98. Hattersley AT, Patel KA. Precision diabetes: learning from monogenic diabetes. Diabetologia. 2017;60:769-777. doi:10.1007/s00125-0174226-2

99. Riddle MC, Philipson LH, Rich SS, et al. Monogenic diabetes: from genetic insights to population-based precision in care. reflections from a diabetes care editors' expert forum. Diabetes Care. 2020;43:3117-3128. doi:10.2337/dci20-0065

100. Carmody D, Naylor RN, Bell CD, et al. GCK-MODY in the US National Monogenic Diabetes Registry: frequently misdiagnosed and unnecessarily treated. Acta Diabetol. 2016;53:703-708. doi:10.1007/s00592-016-0859-8

101. Mahajan A, Taliun D, Thurner M, et al. Fine-mapping type 2 diabetes loci to single-variant resolution using high-density imputation and islet-specific epigenome maps. Nat Genet. 2018;50:1505. doi:10.1038/s41588-018-0241-6

102. Meigs JB. The genetic epidemiology of type 2 diabetes: opportunities for health translation. Curr Diabetes Rep. 2019;19. doi:10.1007/s11892019-1173-y

103. Udler MS, McCarthy MI, Florez JC, Mahajan A. Genetic risk scores for diabetes diagnosis and precision medicine. Endocr Rev. 2019;40:1500-1520. doi:10.1210/er.2019-00088

104. Li JEH, Szczerbinski L, Dawed AY, et al. A polygenic score for type 2 diabetes risk is associated with both the acute and sustained response to sulfonylureas. Diabetes. 2021;70:293-300. doi:10.2337/db20-0530

105. Leighton E, Sainsbury CAR, Jones GC. A practical review of C-Peptide testing in diabetes. Diabetes Ther. 2017;8:475-487. doi:10.1007/ s13300-017-0265-4

106. Kumar S, Subhakumari KN. Role of anti-GAD, anti-IA2 antibodies and C-peptide in differentiating latent autoimmune diabetes in adults from type 2 diabetes mellitus. Int J Diabetes Dev Ctries. 2016;36:313-319. doi:10.1007/s13410-015-0451-8

107. Laakso M. Biomarkers for type 2 diabetes. Mol Metab. 2019;27:S139-S46. doi:10.1016/j.molmet.2019.06.016

Diabetes, Metabolic Syndrome and Obesity: Targets and Therapy

Dovepress

\section{Publish your work in this journal}

Diabetes, Metabolic Syndrome and Obesity: Targets and Therapy is an international, peer-reviewed open-access journal committed to the rapid publication of the latest laboratory and clinical findings in the fields of diabetes, metabolic syndrome and obesity research. Original research, review, case reports, hypothesis formation, expert opinion and commentaries are all considered for publication. The manuscript management system is completely online and includes a very quick and fair peer-review system, which is all easy to use. Visit http://www.dovepress. com/testimonials.php to read real quotes from published authors.

Submit your manuscript here: https://www.dovepress.com/diabetes-metabolic-syndrome-and-obesity-targets-and-therapy-journal 